PAPER

Spectral properties of the two-dimensional Schrödinger Hamiltonian with various solvable confinements in the presence of a central point perturbation

To cite this article: S Fassari et al 2019 Phys. Scr. 94055202

View the article online for updates and enhancements.

\section{Recent citations}

$$
\begin{aligned}
& \text { - The Birman-Schwinger Operator for a } \\
& \text { Parabolic Quantum Well in a Zero- } \\
& \hline \text { Thickness Laver in the Presence of a Two- } \\
& \text { Dimensional Attractive Gaussian Impurity } \\
& \text { Sergio Albeverio et al } \\
& \text { - Point Interactions With Bias Potentials } \\
& \text { Alexander V. Zolotaryuk et al }
\end{aligned}
$$




\title{
Spectral properties of the two-dimensional Schrödinger Hamiltonian with various solvable confinements in the presence of a central point perturbation
}

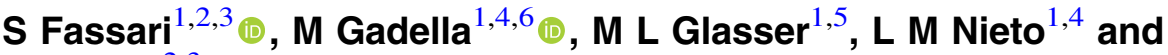 \\ F Rinaldi ${ }^{2,3}$ \\ ${ }^{1}$ Departamento de Física Teórica, Atómica y Óptica, Universidad de Vãlladolid, E-47011 Valladolid, Spain \\ ${ }^{2}$ CERFIM, PO Box 1132, Via F. Rusca 1, CH-6601 Locarno, Switzerland \\ ${ }^{3}$ Dipartimento di Fisica Nucleare, Subnucleare e delle Radiazioni, Universitá degli Studi Guglielmo \\ Marconi, Via Plinio 44, I-00193 Rome, Italy \\ ${ }^{4}$ IMUVA- Instituto de Matematicas, Universidad de Valladolid, E-47011 Valladolid, Spain \\ ${ }^{5}$ Department of Physics, Clarkson University, Potsdam, NY 13699, United States of America \\ E-mail: silvestro.fassari@uva.es, manuelgadella1@gmail.com, laryg@tds.net, luismiguel.nieto.calzada@ \\ uva.es and f.rinaldi@unimarconi.it
}

Received 10 January 2019, revised 1 February 2019

Accepted for publication 8 February 2019

Published 6 March 2019

\begin{abstract}
We study three solvable two-dimensional systems perturbed by a point interaction centered at the origin. The unperturbed systems are the isotropic harmonic oscillator, a square pyramidal potential and a combination thereof. We study the spectrum of the perturbed systems. We show that, while most eigenvalues are not affected by the point perturbation, a few of them are strongly perturbed. We show that for some values of one parameter, these perturbed eigenvalues may take lower values than the immediately lower eigenvalue, so that level crossings occur. These level crossings are studied in some detail.
\end{abstract}

Keywords: point interaction, solvable models, level crossing, confining potentials

(Some figures may appear in colour only in the online journal)

\section{Introduction}

This is a new study of quantum solvable potentials with a point interaction. In the present paper, we shall focus our attention on two-dimensional systems as a continuation of the analysis of one-dimensional systems with point interactions. There is a large number of articles in the scientific literature dealing with point potentials (also called contact potentials and also occasionally singular potentials) in quantum mechanics $[1,2]$. The advantage of one-dimensional models with point perturbations is clear as they are useful for the study of a variety of qualitative properties [3-13]. They have been used to model several kinds of extra thin structures $[14,15]$, to mimic point defects in

${ }^{6}$ Author to whom any correspondence should be addressed. materials, or to study heterostructures $[16,17]$. In any case, point potentials may serve as solvable or quasi solvable models that approximate results for very short range potentials. Conversely, point potentials may be sometimes written as limits of very short range potentials $[1,18,19]$. Point potentials play an interesting role as impurities in quantum field theory models (as one may see in [20-23]) and in a recent reinterpretation of the Casimir effect [24], and may also have unexpected relations with other fields like group theory [25].

Point potentials have been also widely investigated in three dimensions [24, 26], where renormalisation is required [1, 2]. Some authors have also considered contact potentials supported by two-dimensional manifolds such as spheres [27], although in the present work we deal with contact potentials supported at the origin only [1, 2]. The analysis of two-dimensional point 
potentials may have an interest in the study of impurities or quantum dots in quantum systems of the form of thin layers such as the graphene. Point potentials show differences depending on the dimension of the ambient space. Differences between physical results concerning very short range interactions in terms of the dimension of the ambient space have been studied in terms of spectral properties [28] or the relations between the energy shifts and the scattering lengths [29]. These differences depend on the physical system under consideration [30]. We wish to remind the reader that a remarkable spectral difference arises between the three-dimensional case and its two-dimensional counterpart in the absence of a confining potential when the free Hamiltonian is simply given by the negative Laplacian: while in the first case the unique negative eigenvalue exists only if the point perturbation is attractive, in the latter case it exists even if the point perturbation is repulsive [1].

In the present study, we often want to obtain data concerning bound states or resonances for which Green functions may be very useful. Now, point interactions quite often produce infinities affecting these Green functions, which are generally removable with the proper use of regularisation techniques [31], which in addition guarantee the self-adjointness of the total Hamiltonian. These regularisation techniques may also be necessary for one-dimensional systems [3, 4, 6, 8, 32].

In the present paper, we are considering a two-dimensional Dirac delta interaction supported at the origin in two different situations. In the former, the Dirac delta decorates an isotropic two-dimensional harmonic oscillator, so that the total Hamiltonian is

$$
\begin{aligned}
H_{\alpha}^{o} & =H_{0}^{o}-\frac{1}{\alpha} \delta(\mathbf{x})=\frac{1}{2}\left(-\Delta+x^{2}+y^{2}\right)-\frac{1}{\alpha} \delta(\mathbf{x}) \\
& =h_{0}^{o} \otimes I+I \otimes h_{0}^{o}-\frac{1}{\alpha} \delta(\mathbf{x}),
\end{aligned}
$$

where $h_{0}^{o}=\frac{1}{2}\left(-\frac{\mathrm{d}^{2}}{\mathrm{~d} x^{2}}+x^{2}\right)$ is the one-dimensional harmonic oscillator Hamiltonian and $\mathbf{x}=(x, y)$. Here, the solution of the eigenvalue problem for the harmonic oscillator is well known, including asymptotic properties [33, 5, 34-37]. When switching on the delta perturbation, we observe that the energy values remain unaltered with two exceptions: the ground state and the $s$-wave for some eigenvalues characterised by two even indices. Those eigenvalues have a strong dependence on the parameter $\alpha$ and, except for the ground state, cross the precedent lower eigenvalue, implying the presence of energy crossings. The levels of the one-dimensional harmonic oscillator with a potential of the type $-\alpha \delta(x)+\beta \delta^{\prime}(x)$ were considered in [9].

In the second case, we consider a square pyramidal potential for the unperturbed Hamiltonian, so that the total Hamiltonian takes the form

$$
\begin{aligned}
H_{\alpha}^{p} & =H_{0}^{p}-\frac{1}{\alpha} \delta(\mathbf{x})=\frac{1}{2}(-\Delta+|x|+|y|)-\frac{1}{\alpha} \delta(\mathbf{x}) \\
& =h_{0}^{c} \otimes I+I \otimes h_{0}^{c}-\frac{1}{\alpha} \delta(\mathbf{x}),
\end{aligned}
$$

where $h_{0}^{c}=\frac{1}{2}\left(-\frac{\mathrm{d}^{2}}{\mathrm{~d} x^{2}}+|x|\right)$ is the one-dimensional conic Hamiltonian. This is an obvious generalisation to two dimensions of the one-dimensional conic potential with a delta of the form $-\mathrm{d}^{2} / \mathrm{d} x^{2}+|x|-\alpha^{-1} \delta(x)$, studied in $[32,38]$. Knowledge of this one-dimensional case makes the study of the two-dimensional problem under our consideration rather easy. Now, the properties of the eigenvalues for $H_{\alpha}$ are somehow similar to the previous case, although under different circumstances that will be discussed in this article. The presence of level crossings is also observed.

Both situations require normalisation in order to have a rigorously defined Green function and, as a consequence, a self-adjoint total Hamiltonian $H_{\alpha}^{o}$ or $H_{\alpha}^{p}$. We have made use of the simple cut-off regularisation procedure, which is sufficient here for our purposes.

A third possibility is a mixed model in which one coordinate behaves as an oscillator and the other as a conic potential. The total Hamiltonian for this situation is

$$
\begin{aligned}
H_{\alpha}^{M} & :=H_{0}^{M}-\frac{1}{\alpha} \delta(\mathbf{x})=\frac{1}{2}\left(-\Delta+x^{2}+|y|\right)-\frac{1}{\alpha} \delta(\mathbf{x}) \\
& =h_{0}^{o} \otimes I+I \otimes h_{0}^{c}-\frac{1}{\alpha} \delta(\mathbf{x}) .
\end{aligned}
$$

The superscript $M$ stands for 'mixed' and the role of the variables $x$ and $y$ may be interchanged, without any relevant modification.

One may also consider some other types of two-dimensional unperturbed Hamiltonians $H_{0}=-\Delta+V$, where $V$ is one of the following potentials:

$$
\begin{aligned}
& V_{1}=\sqrt{x^{2}+y^{2}}, \quad V_{2}=|x|^{a}+|y|^{b}, \quad 0<a, b, \\
& V_{3}=\frac{-1}{\sqrt{x^{2}+y^{2}}} .
\end{aligned}
$$

The first potential in (1.4) may look like the straightforward generalisation of the conic potential $V=|x|$. However, the solution of the eigenvalue problem for this Hamiltonian does not seem easy even after the choice of polar coordinates. The third example has been considered in [39, 40]. In any case, their study is left for further work.

We are organising the present article as follows: in section 2, we study the two-dimensional isotropic harmonic oscillator with a delta. In section 3, we make an analogous analysis for the two-dimensional pyramidal potential. We discuss the mixed case in section 4 . We close this article with some concluding remarks plus a brief appendix with several mathematical results related with the convergence of some series relevant for our discussion.

\section{Two-dimensional harmonic oscillator plus a contact potential}

The objective of the present section is the study of the spectral properties of the isotropic two-dimensional harmonic oscillator (1.1). Previous experiences with one and three dimensions [9, 33], show that the use of the Green function gives an excellent method for the determination of the eigenvalues associated to these types of Hamiltonians. As is well known, 
the Green function associated to some self-adjoint operator $H$ is the kernel of the resolvent operator, $(H-E)^{-1}$ associated to $H$ and will be denoted as $(H-E)^{-1}(\mathbf{x}, \mathbf{y})$ along the present manuscript. Since our configuration space is twodimensional, both variables $(\mathbf{x}, \mathbf{y})$ in our Green functions are two-dimensional. When $H$ has a purely discrete spectrum, the Green function for $H$ is given by

$$
G(\mathbf{x}, \mathbf{y})=(H-E)^{-1}(\mathbf{x}, \mathbf{y})=\sum_{\mathbf{n}} \frac{\psi_{\mathbf{n}}(\mathbf{x}) \psi_{\mathbf{n}}(\mathbf{y})}{E_{\mathbf{n}}-E},
$$

where $H \psi_{\mathbf{n}}(\mathbf{x})=E_{\mathbf{n}} \psi_{n}(\mathbf{x})$ and the sum extends to all possible bound states of $H$. Properties of the integral operator defined by the kernel (2.1) will be analysed in the mathematical appendix at the end of this paper.

We begin our discussion with the consideration of some properties of the Green function $G_{0}(\mathbf{x}, \mathbf{y})$ for the operator $H_{0}^{o}$ as defined in (1.1) (For a discussion on the Green function for the harmonic oscillator in any dimension, see [41]). As $H_{0}^{o}$ is the isotropic two-dimensional harmonic oscillator, the energy levels are given by $E_{n_{1}, n_{2}}=\left(n_{1}+n_{2}+1\right)$ (for convenience, we have chosen from the very beginning units such that $\hbar \omega=1)$. We denote the corresponding eigenfunctions as $\psi_{n_{1}, n_{2}}(\mathbf{x})=\phi_{n_{1}}\left(x_{1}\right) \phi_{n_{2}}\left(x_{2}\right)$, where $\phi_{n}(z)$ are the onedimensional normalised Hermite functions. Then, we have our first result:

Theorem 2.1. The function $\left(H_{0}^{o}-E\right)^{-1}(\mathbf{x}, \mathbf{0})$ belongs to $L^{2}\left(\mathbb{R}^{2}\right)$ for any $\mathrm{E}$ in the resolvent set $\rho\left(H_{0}^{o}\right)$.

Proof. As a consequence of the first resolvent identity, see [42] page 198, we need only prove the statement for any $E<0$. The square of the $L^{2}$-norm of the function $\left(H_{0}^{o}-E\right)^{-1}(\mathbf{x}, \mathbf{0})$ can be expressed as follows:

$$
\begin{aligned}
& \left\|\left(H_{0}^{o}-E\right)^{-1}(\mathbf{x}, \mathbf{0})\right\|_{2}^{2} \\
& =\left\|\sum_{n_{1}=0}^{\infty} \sum_{n_{2}=0}^{\infty} \frac{\psi_{n_{1}, n_{2}}\left(x_{1}, x_{2}\right) \psi_{n_{1}, n_{2}}(0,0)}{n_{1}+n_{2}+1-E}\right\|^{2} \\
& =\sum_{n_{1}=0}^{\infty} \sum_{n_{2}=0}^{\infty} \frac{\left|\psi_{n_{1}, n_{2}}(0,0)\right|^{2}}{\left|n_{1}+n_{2}+1-E\right|^{2}},
\end{aligned}
$$

where the last identity is a consequence that $\psi_{n_{1}, n_{2}}(\mathbf{x}), n_{1}, n_{2}=$ $0,1,2, \ldots$ is an orthonormal basis in $L^{2}\left(\mathbb{R}^{2}\right)$. After $\psi_{n_{1}, n_{2}}(\mathbf{x})=$ $\phi_{n_{1}}\left(x_{1}\right) \phi_{n_{2}}\left(x_{2}\right)$ and taking into account that Hermite functions of odd index are odd functions, so that they vanish at zero, the last expression in (2.2) is equal to

$$
\begin{aligned}
& \sum_{n_{1}=0}^{\infty} \sum_{n_{2}=0}^{\infty} \frac{\phi_{2 n_{1}}^{2}(0) \phi_{2 n_{2}}^{2}(0)}{\left(2 n_{1}+2 n_{2}+1-E\right)^{2}} \\
& \quad<\sum_{n_{1}=0}^{\infty} \sum_{n_{2}=0}^{\infty} \frac{\phi_{2 n_{1}}^{2}(0) \phi_{2 n_{2}}^{2}(0)}{\left(2 n_{1}+2 n_{2}+1\right)^{2}}=\left\|\left(H_{0}^{o}\right)^{-1}(\mathbf{x}, \mathbf{0})\right\|_{2}^{2} \\
& \quad<\left[\sum_{n=0}^{\infty} \frac{\phi_{2 n}^{2}(0)}{2 n+1}\right]^{2}=\left[\frac{1}{\sqrt{\pi}} \int_{0}^{\infty} \frac{e^{-t} d t}{\left(1-e^{-2 t}\right)^{\frac{1}{2}}}\right]^{2}=\frac{\pi}{4},
\end{aligned}
$$

where the first inequality in the first line of (2.3) comes after $E<0$ and the first identity in the second line has been shown in [34]. This proves our assertion.
A general result shows that $G(\mathbf{x}, \mathbf{y})$ is in $L^{2}\left(\mathbb{R}^{2}\right)$ as a function of $\mathbf{x}$ for almost all $\mathbf{y} \in \mathbb{R}^{2}$ and the same as a function of $\mathbf{y}$ for almost all $\mathbf{x} \in \mathbb{R}^{2}$, see [43] page 102. Here, we have proved that the origin in $\mathbb{R}^{2}$ is among the points at which $G^{o}(\mathbf{x}, \mathbf{0}) \in L^{2}\left(\mathbb{R}^{2}\right)$. The same holds with respect to $G^{o}(\mathbf{0}, \mathbf{y})$.

Another general result [34] shows that for any real $\alpha \neq 0$, the resolvent of $H_{\alpha}^{o}$ is given by the following expression

$$
\begin{aligned}
G_{\alpha}(\mathbf{x}, \mathbf{y})= & \left(H_{\alpha}^{o}-E\right)^{-1}=\left(H_{0}^{o}-E\right)^{-1} \\
& +\frac{\left|\left(H_{0}^{o}-E\right)^{-1}(\mathbf{x}, \mathbf{0})\right\rangle\left\langle\left(H_{0}^{o}-E\right)^{-1}(\mathbf{0}, \mathbf{y})\right|}{\alpha-\left(H_{0}^{o}-E\right)^{-1}(\mathbf{0}, \mathbf{0})}
\end{aligned}
$$

where $H_{\alpha}^{o}$ has been defined in (1.1).

In the denominator in (2.4), we have to use the value of the Green function for the free Hamiltonian at the point $(\mathbf{0}, \mathbf{0}) \in \mathbb{R}^{4},\left(H_{0}^{o}-E\right)^{-1}(\mathbf{0}, \mathbf{0})$, which is formally given by

$$
\begin{aligned}
& \left(H_{0}^{o}-E\right)^{-1}(\mathbf{0}, \mathbf{0}) \\
& \quad=\sum_{n_{1}=0}^{\infty} \sum_{n_{2}=0}^{\infty} \frac{\phi_{2 n_{1}}^{2}(0) \phi_{2 n_{2}}^{2}(0)}{2 n_{1}+2 n_{2}+1-E} .
\end{aligned}
$$

Although $\left(H_{0}^{o}-E\right)^{-1}(\mathbf{x}, \mathbf{0})$ is a square integrable function, its value at the origin is not finite, as the series in (2.5) does not converge in general. This means that we need a renormalisation procedure in order to obtain the resolvent for a selfadjoint determination for $H_{\alpha}^{o}$. To this end, let us replace $\left(H_{0}^{o}-E\right)^{-1}(\mathbf{0}, \mathbf{0})$ by means of the following limit:

$$
\lim _{N \rightarrow \infty}\left[\sum_{n_{1}=0}^{N} \sum_{n_{2}=0}^{N} \frac{\phi_{2 n_{1}}^{2}(0) \phi_{2 n_{2}}^{2}(0)}{2 n_{1}+2 n_{2}+1-E}-\frac{1}{\mu(N)}\right],
$$

with

$$
\frac{1}{\mu(N)}=\sum_{n_{1}=0}^{N} \sum_{n_{2}=0}^{N} \frac{\phi_{2 n_{1}}^{2}(0) \phi_{2 n_{2}}^{2}(0)}{2 n_{1}+2 n_{2}+1} .
$$

This is the so called cut-off renormalisation. After this procedure, we finally obtain

$$
\begin{aligned}
& \left(H_{\alpha}^{o}-E\right)^{-1}=\left(H_{0}^{o}-E\right)^{-1} \\
& +\frac{\left|\left(H_{0}^{0}-E\right)^{-1}(\mathbf{x}, \mathbf{0})\right\rangle\left\langle\left(H_{0}^{0}-E\right)^{-1}(\mathbf{0}, \mathbf{y})\right|}{\alpha-E \sum_{n_{1}=0}^{\infty} \sum_{n_{2}=0}^{\infty} \frac{\phi_{2 n_{1}}^{2}(0) \phi_{2 n_{2}}^{2}(0)}{\left(2 n_{1}+2 n_{2}+1\right)\left(2 n_{1}+2 n_{2}+1-E\right)}} .
\end{aligned}
$$

Note that (2.7) is a truncated version of $\left(H_{0}^{o}\right)^{-1}(\mathbf{0}, \mathbf{0})$, replacing the sum up to infinite by the sum up to $N$. Observe that $\left(H_{\alpha}^{o}-E\right)^{-1}$ is the sum of the resolvent of the free Hamiltonian $H_{0}^{o}$ plus a rank one operator and, therefore, it is a perfectly defined bounded operator. We still need to show that this is the resolvent of a self-adjoint operator. This proof is essentially identical to the proof for the three-dimensional case given in [33] or even for the case of a negative threedimensional Laplacian perturbed by a Dirac delta given in [1] and, therefore, we omit it. The conclusion can be summarised as follows:

Theorem 2.2. The bounded operator $\left(H_{\alpha}^{o}-E\right)^{-1}$ is the resolvent of the self-adjoint Hamiltonian, which is usually 
given by the heuristic expression $H_{\alpha}^{o}=H_{0}-\mu_{\alpha}|\delta\rangle\langle\delta|$ with

$$
\begin{aligned}
\mu_{\alpha} & =\left[\sum_{n_{1}=0}^{\infty} \sum_{n_{2}=0}^{\infty} \frac{\phi_{2 n_{1}}^{2}(0) \phi_{2 n_{2}}^{2}(0)}{\left(2 n_{1}+2 n_{2}+1\right)}+\alpha\right]^{-1} \\
& =\left[\left(H_{0}^{o}\right)^{-1}(\mathbf{0}, \mathbf{0})+\alpha\right]^{-1}, \quad \alpha \in \mathbb{R} .
\end{aligned}
$$

Moreover, the family of Hamiltonians $H_{\alpha}^{o}$ is an analytic family with respect to $\alpha$ in the sense of Kato [1].

Before going further, we wish to stress that $H_{\alpha=0}^{o} \neq H_{0}^{o}$ and $H_{\alpha=\infty}^{o}=H_{0}^{o}$ as we may easily deduce from (2.8).

An important remark concerning the eigenvalue multiplicity: As is well known, the multiplicity of the eigenvalue $E_{n, m}=n+m+1$ of $H_{0}^{o}$ is precisely $n+m+1$. If either $m$ or $n$ are odd, then, $E_{m, n}$ is an eigenvalue of $H_{\alpha}^{o}$ with identical degeneracy $n+m+1$, as the perturbation does not affect to the eigenspace of $E_{n, m}$. Furthermore, although $E_{2 n, 2 m}=2 n+$ $2 m+1$ for $n, m>1$ is still in the spectrum of $H_{\alpha}^{o}$, it has multiplicity $2 n+2 m$ due to the emergence of a new eigenvalue. Its eigenvector was in the eigenspace of $H_{0}^{o}$ with eigenvalue $E_{2 n, 2 m}$.

The eigenvalues of $H_{\alpha}^{o}$ are the zeroes of the denominator in (2.8). The ground state energy and the new eigenvalues of the operator $H_{\alpha}^{o}$ are given by the solutions on $E$ of the equation:

$\alpha=E \sum_{n_{1}=0}^{\infty} \sum_{n_{2}=0}^{\infty} \frac{\phi_{2 n_{1}}^{2}(0) \phi_{2 n_{2}}^{2}(0)}{\left(2 n_{1}+2 n_{2}+1\right)\left(2 n_{1}+2 n_{2}+1-E\right)}$.

It is interesting to write an integral expression for the series on the right hand side of (2.10) valid for $E<1$. To begin with and due to (2.1), we can easily show that the right hand side in (2.10) is just equal to $\left(H_{0}^{o}-E\right)^{-1}(\mathbf{0}, \mathbf{0})-\left(H_{0}^{o}\right)^{-1}(\mathbf{0}, \mathbf{0})$, so that

$$
\begin{aligned}
\alpha= & \sum_{n_{1}=0}^{\infty} \sum_{n_{2}=0}^{\infty} \frac{\phi_{2 n_{1}}^{2}(0) \phi_{2 n_{2}}^{2}(0)}{2 n_{1}+2 n_{2}+1-E} \\
& -\sum_{n_{1}=0}^{\infty} \sum_{n_{2}=0}^{\infty} \frac{\phi_{2 n_{1}}^{2}(0) \phi_{2 n_{2}}^{2}(0)}{2 n_{1}+2 n_{2}+1} \\
= & \sum_{n_{1}=0}^{\infty} \sum_{n_{2}=0}^{\infty} \phi_{2 n_{1}}^{2}(0) \phi_{2 n_{2}}^{2}(0) \\
& \times \int_{0}^{\infty} \mathrm{e}^{-\left(2 n_{1}+2 n_{2}+1\right) t}\left(\mathrm{e}^{E t}-1\right) \mathrm{d} t \\
= & \int_{0}^{\infty} \sum_{n_{1}=0}^{\infty} \sum_{n_{2}=0}^{\infty} \phi_{2 n_{1}}^{2}(0) \phi_{2 n_{2}}^{2}(0) \\
& \times \mathrm{e}^{-\left(2 n_{1}+2 n_{2}+1\right) t}\left(\mathrm{e}^{E t}-1\right) \mathrm{d} t \\
= & \int_{0}^{\infty}\left[\sum_{n=0}^{\infty} \phi_{2 n}^{2}(0) \mathrm{e}^{-(2 n+1 / 2) t}\right]^{2}\left(\mathrm{e}^{E t}-1\right) \mathrm{d} t,
\end{aligned}
$$

where the identity between the second and the third line in (2.11) is due to the Lebesgue convergence theorem (see [33]). The sum of the expression between brackets in the last integral in (2.11) was obtained in [34]. It is the value of $\left(H_{0}^{o}-E\right)^{-1}(\mathbf{0}, \mathbf{0})$ with $E=0$, where $H_{0}^{o}$ is now the one-dimensional harmonic oscillator. Then, (2.11) is given by

$$
\begin{aligned}
\alpha & =\frac{1}{\pi} \int_{0}^{\infty} \frac{\mathrm{e}^{t}\left(\mathrm{e}^{E t}-1\right)}{\mathrm{e}^{2 t}-1} \mathrm{~d} t \\
& =\frac{1}{\pi} \int_{0}^{\infty} \frac{\mathrm{e}^{-t}\left(\mathrm{e}^{E t}-1\right)}{1-\mathrm{e}^{-2 t}} \mathrm{~d} t \\
& =\frac{1}{\pi} \int_{0}^{1} \frac{\xi^{-E}-1}{1-\xi^{2}} \mathrm{~d} \xi,
\end{aligned}
$$

with $\xi=\mathrm{e}^{-t}$. We need the condition $E<1$ in order to guarantee the convergence of the integral in (2.12). We wish to transform this expression into a more workable one. We start with a simple manipulation in the last integral in (2.12), so that

$$
\begin{aligned}
\alpha & =\frac{1}{2 \pi}\left[2 \int_{0}^{1} \frac{\xi^{-E} \mathrm{~d} \xi}{1-\xi^{2}}-2 \int_{0}^{1} \frac{\mathrm{d} \xi}{1-\xi^{2}}\right] \\
& =\frac{1}{2 \pi} \lim _{\varepsilon \rightarrow 0_{+}}\left[2 \int_{0}^{1} \frac{\xi^{-E} \mathrm{~d} \xi}{\left(1-\xi^{2}\right)^{1-\varepsilon}}-2 \int_{0}^{1} \frac{\mathrm{d} \xi}{\left(1-\xi^{2}\right)^{1-\varepsilon}}\right]
\end{aligned}
$$

Using the properties of the Gamma function, we may write (2.13) as

$\alpha=\frac{1}{2 \pi} \lim _{\varepsilon \rightarrow 0_{+}} \Gamma(\varepsilon)\left[\frac{\Gamma(1 / 2-E / 2)}{\Gamma(1 / 2-E / 2+\varepsilon)}-\frac{\Gamma(1 / 2)}{\Gamma(1 / 2+\varepsilon)}\right]$.

Next, we use in (2.14) some well-known relations, namely:

$$
\begin{aligned}
& \Gamma(\varepsilon) \Gamma(1-\varepsilon)=\frac{\pi}{\sin (\pi \varepsilon)}, \quad \lim _{\varepsilon \rightarrow 0_{+}} \frac{\sin (\pi \varepsilon)}{\varepsilon}=\pi \\
& \text { and } \lim _{\varepsilon \rightarrow 0_{+}} \Gamma(1-\varepsilon)=1,
\end{aligned}
$$

so that (2.14) yields

$$
\begin{aligned}
\alpha= & \frac{1}{2 \pi} \lim _{\varepsilon \rightarrow 0_{+}} \frac{1}{\Gamma\left(\frac{1}{2}+\varepsilon\right) \Gamma\left(\frac{1}{2}-E / 2+\varepsilon\right)} \\
& \times\left[\frac{\Gamma\left(\frac{1}{2}-E / 2\right) \Gamma\left(\frac{1}{2}+\varepsilon\right)-\Gamma\left(\frac{1}{2}-E / 2+\varepsilon\right) \Gamma\left(\frac{1}{2}\right)}{\varepsilon}\right] \\
= & \frac{1}{2 \pi} \frac{1}{\Gamma\left(\frac{1}{2}\right) \Gamma\left(\frac{1}{2}-E / 2\right)} \\
& \times \lim _{\varepsilon \rightarrow 0_{+}}\left[\frac{\Gamma\left(\frac{1}{2}-E / 2\right) \Gamma\left(\frac{1}{2}+\varepsilon\right)-\Gamma\left(\frac{1}{2}-E / 2+\varepsilon\right) \Gamma\left(\frac{1}{2}\right)}{\varepsilon}\right] \\
= & \left.\frac{1}{2 \pi} \frac{1}{\Gamma(1 / 2) \Gamma(1 / 2-E / 2)}\right] \\
& \times\left[\Gamma(1 / 2-E / 2) \Gamma^{\prime}(1 / 2)-\Gamma(1 / 2) \Gamma^{\prime}(1 / 2-E / 2)\right] \\
= & \frac{1}{2 \pi}\left[\frac{\Gamma^{\prime}(1 / 2)}{\Gamma(1 / 2)}-\frac{\Gamma^{\prime}(1 / 2-E / 2)}{\Gamma(1 / 2-E / 2)}\right] \\
= & \frac{1}{2 \pi}\left[(\ln \Gamma)^{\prime}(1 / 2)-(\ln \Gamma)^{\prime}(1 / 2-E / 2)\right],
\end{aligned}
$$

where the prime denotes the derivative of the Gamma function in its argument. One well-known relation is

$$
\psi(x)=(\ln \Gamma(x))^{\prime}=-\gamma-\sum_{n=1}^{\infty}\left(\frac{1}{n+x-1}-\frac{1}{n}\right),
$$



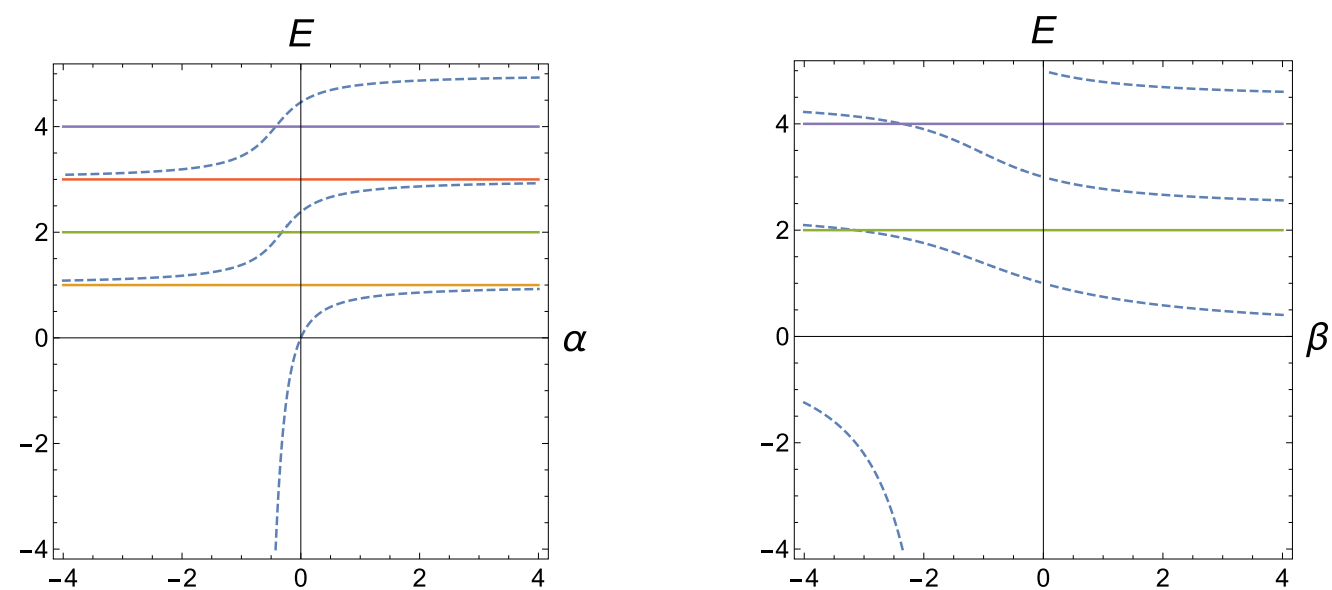

Figure 1. Plots of the three lowest eigenvalues from (2.19) created by the point perturbation: on the left as functions of the extension parameter $\alpha$, on the right as functions of the strength parameter $\beta$ (the horizontal lines are those of the unperturbed oscillator which, with the exclusion of $E_{0}=1$, are still in the spectrum).

where $\gamma$ is the Euler-Mascheroni constant, $\gamma \approx 0.577216$. If we use (2.17) in the last expression in (2.16), we readily obtain that

$$
\begin{aligned}
\alpha & =\frac{1}{2 \pi}[\psi(1 / 2)-\psi(1 / 2-E / 2)] \\
& =\frac{E}{4 \pi} \sum_{n=1}^{\infty} \frac{1}{\left(n-\frac{1}{2}\right)\left(n-\frac{1+E}{2}\right)},
\end{aligned}
$$

which is essentially our final formula. By setting $\beta:=1 / \alpha$, this can be also written as

$$
\begin{aligned}
\alpha & =\frac{1}{\beta}=\frac{E}{4 \pi} \sum_{n=0}^{\infty} \frac{1}{\left(n+\frac{1}{2}\right)\left(n+\frac{1-E}{2}\right)} \\
& =-\frac{\gamma+\ln 4+\psi((1-E) / 2)}{2 \pi} .
\end{aligned}
$$

In particular, for any $E$ given by a positive even number, the total energy of an antisymmetric state, the series on the right hand side becomes a simple telescoping series so that it is rather straightforward to find the location of the points where level crossings occur. For the lowest antisymmetric level with $E=2$ we find $\beta_{1}=-\pi$, whereas for the upper one $E=4$ we have $\beta_{2}=-\frac{3}{4} \pi$. The plot of the lowest eigenvalues as functions of the extension parameter $\alpha$ is shown on the left hand side of figure 1. Alternatively, the right hand side of figure 1 depicts the perturbed eigenvalues as functions of the parameter representing the strength of the point interaction $(\beta)$.

The striking difference between the above graphs and either their three-dimensional analogues in $[44,45]$ or those of the one-dimensional harmonic (respectively conic) oscillator perturbed by the $\left|\delta^{\prime}\right\rangle\left\langle\delta^{\prime}\right|$-interaction investigated in [3] (respectively $[32,38]$ ) is that the level crossings do not occur at the same point and, furthermore, are located along the negative semi-axis. Hence, it is reasonable to conjecture that the difference may arise because of the logarithmic divergences which are a peculiar feature of two-dimensional quantum mechanics.

\section{The square pyramidal potential with a contact perturbation}

One of the possible generalisations of the one-dimensional conic potential to higher dimensions is the square pyramidal potential. If we restrict ourselves to two dimensions and make a choice of units to simplify the form of the Hamiltonian, we have the expression (1.2) for the two-dimensional pyramidal Hamiltonian $H_{0}^{p}$.

$$
\begin{aligned}
H_{0}^{p} & :=\frac{1}{2}(-\Delta+|x|+|y|)=\frac{1}{2}\left[h_{0}^{c} \otimes I+I \otimes h_{0}^{c}\right], \\
h_{0}^{c} & =\frac{1}{2}\left[-\frac{\mathrm{d}^{2}}{\mathrm{~d} x^{2}}+|x|\right] .
\end{aligned}
$$

As a consequence of theorem VIII.3, its corollary and example 1 in [42], the operator $H_{0}^{p}$ is essentially self-adjoint, i.e. it has a unique self-adjoint extension, on $D\left(h_{0}^{c}\right) \otimes D\left(h_{0}^{c}\right)$, where $D\left(h_{0}^{c}\right)$ is the domain on which $h_{0}^{c}$ is self-adjoint. Note that $H_{0}^{p}$ is an obvious generalisation of $h_{0}^{c}$. The spectral properties of this one-dimensional conic Hamiltonian decorated with a point impurity have been studied in [32], see also $[38,47]$. Thus, we may use the results in $[32,38]$ concerning eigenvalues and eigenfunctions of the one-dimensional conic oscillator in order to write the resolvent of the operator $H_{0}^{p}$ as in (3.1) to conclude that

$$
\left(H_{0}^{p}-E\right)^{-1}=\sum_{n=1}^{\infty} \sum_{m=1}^{\infty} \frac{\left|\psi_{n} \otimes \psi_{m}\right\rangle\left\langle\psi_{n} \otimes \psi_{m}\right|}{E_{n}+E_{m}-E},
$$

where the eigenvalues $E_{n}$ are the solutions of the following transcendental equations

$$
\begin{cases}A i^{\prime}\left(-2 E_{2 n-1}\right)=0, & n=1,2, \ldots \\ A i\left(-2 E_{2 n}\right)=0, & n=1,2, \ldots\end{cases}
$$

where $\operatorname{Ai}(x)$ represents the Airy function and the prime gives the derivative of the Airy function with respect to its argument. The corresponding normalised eigenfunctions are 
given by

$$
\begin{aligned}
\psi_{2 n-1}(x) & =\frac{A i\left(|x|-2 E_{n-1}\right)}{2 \sqrt{E_{2 n-1}} A i\left(-2 E_{2 n-1}\right)}, \quad n=1,2, \ldots \\
\psi_{2 n}(x) & =\frac{\operatorname{sgn}(x) A i\left(|x|-2 E_{2 n}\right)}{\sqrt{2} A i^{\prime}\left(-2 E_{2 n}\right)}, \quad n=1,2, \ldots
\end{aligned}
$$

where $\operatorname{sgn}(x)$ denotes the sign function. Properties of the integral operator defined by (3.2) will be analysed in the appendix at the end of the paper.

Now, we need to give an expression for the resolvent of the Hamiltonian $H_{\alpha}^{p}$. This is just given by replacing $H_{0}^{o}$ by $H_{0}^{p}$ in (2.4). With this idea in mind, we can prove the analogues of theorems 2.1 and 2.2 in this case. The first result is the following:

Theorem 3.1. The function $\left(H_{0}^{p}-E\right)^{-1}(\mathbf{x}, \mathbf{0})$ belongs to $L^{2}\left(\mathbb{R}^{2}\right)$ for any $\mathrm{E}$ in the resolvent set $\rho\left(H_{0}^{p}\right)$.

Proof. As a consequence of the first resolvent identity [42], we need only prove the statement for any $E<0$. The square of the $L^{2}$-norm of the function $\left(H_{0}^{p}-E\right)^{-1}(\mathbf{x}, \mathbf{0})$ can be expressed as follows:

$$
\begin{aligned}
& \left\|\left(H_{0}^{p}-E\right)^{-1}(\mathbf{x}, \mathbf{0})\right\|_{2}^{2} \\
& \quad=\sum_{n=1}^{\infty} \sum_{m=1}^{\infty} \frac{\psi_{2 m-1}^{2}(0) \psi_{2 n-1}^{2}(0)}{\left(E_{2 n-1}+E_{2 m-1}-E\right)^{2}} \\
& \quad \leqslant \sum_{n=1}^{\infty} \sum_{m=1}^{\infty} \frac{\psi_{2 m-1}^{2}(0) \psi_{2 n-1}^{2}(0)}{\left(E_{2 n-1}+E_{2 m-1}\right)^{2}}=\left\|\left(H_{0}^{p}\right)^{-1}(\mathbf{x}, \mathbf{0})\right\|_{2}^{2} .
\end{aligned}
$$

The series in the second row of (3.5) converges. This is due to the fact that the sequence $E_{2 n-1}^{-1}$ behaves like $n^{-\frac{2}{3}}$ as $n \rightarrow \infty$, as proved in corollary 3.6 in [46]. See also [32, 38]. It follows that

$$
\begin{aligned}
& \sum_{n=1}^{\infty} \sum_{m=1}^{\infty} \frac{\psi_{2 m-1}^{2}(0) \psi_{2 n-1}^{2}(0)}{\left(E_{2 n-1}+E_{2 m-1}\right)^{2}} \\
& \quad \leqslant \frac{1}{2} \sum_{n=1}^{\infty} \sum_{m=1}^{\infty} \frac{\psi_{2 m-1}^{2}(0) \psi_{2 n-1}^{2}(0)}{E_{2 n-1} E_{2 m-1}} \\
& \quad \leqslant \frac{1}{2}\left[\sum_{n=1}^{\infty} \frac{\psi_{2 n-1}^{2}(0)}{E_{2 n-1}}\right]^{2}=\frac{1}{2}\left[\frac{1}{4} \sum_{n=1}^{\infty} \frac{1}{E_{2 n-1}^{2}}\right]^{2}<\infty
\end{aligned}
$$

This completes the proof.

Our next objective is the construction of a self-adjoint determination for the operator $H_{0}^{p}$ as in (3.1) decorated with a Dirac delta perturbation supported at the origin. We shall achieve it by constructing the resolvent operator associated to this self-adjoint operator, which, in addition, will give the behaviour of the eigenvalues of the perturbed Hamiltonian. As in the previous case and for similar reasons, we advocate a cut-off renormalisation with

$$
\begin{aligned}
\frac{1}{\mu(N)} & =\sum_{n=1}^{N} \sum_{m=1}^{N} \frac{\psi_{2 m-1}^{2}(0) \psi_{2 n-1}^{2}(0)}{E_{2 m-1}+E_{2 n-1}} \\
& =\left(H_{0}^{p N}\right)^{-1}(\mathbf{0}, \mathbf{0}) .
\end{aligned}
$$

Here, the integer $N$ denotes the ultraviolet energy cut-off. After similar operations as done in the previous section, we obtain for $E<0$ the following expression for the resolvent $\left(H_{\alpha}^{p}-E\right)^{-1}$ :

$$
+\frac{\left(H_{0}^{p}-E\right)^{-1}}{\alpha+\lim _{N \rightarrow+\infty}\left[\sum_{m, n=1}^{N} \frac{\psi_{2 m-1}^{2}(0) \psi_{2 n-1}^{2}(0)}{E_{2 m-1}+E_{2 n-1}}-\sum_{m, n=1}^{N} \frac{\psi_{2 m-1}^{2}(0) \psi_{2 n-1}^{2}(0)}{E_{2 m-1}+E_{2 n-1}-E}\right]} .
$$

This expression can be rewritten as

$$
\begin{aligned}
& \left(H_{0}^{p}-E\right)^{-1} \\
& +\frac{\left|\left(H_{0}^{p}-E\right)^{-1}(\mathbf{x}, \mathbf{0})\right\rangle\left\langle\left(H_{0}^{p}-E\right)^{-1}(\mathbf{0}, \mathbf{y})\right|}{\alpha-E \lim _{N \rightarrow+\infty} \sum_{n=1}^{N} \sum_{m=1}^{N} \frac{\psi_{2 m-1}^{2}(0) \psi_{2 n-1}^{2}(0)}{\left(E_{2 m-1}+E_{2 n-1}\right)\left(E_{2 m-1}+E_{2 n-1}-E\right)}},
\end{aligned}
$$

or, more explicitly,

$$
\begin{aligned}
& \left(H_{0}^{p}-E\right)^{-1} \\
& +\frac{\left|\left(H_{0}^{p}-E\right)^{-1}(\mathbf{x}, \mathbf{0})\right\rangle\left\langle\left(H_{0}^{p}-E\right)^{-1}(\mathbf{0}, \mathbf{y})\right|}{\alpha-\frac{E}{16} \sum_{n=1}^{\infty} \sum_{m=1}^{\infty} \frac{1}{E_{2 m-1}\left(E_{2 m-1}+E_{2 n-1}\right)\left(E_{2 m-1}+E_{2 n-1}-E\right) E_{2 n-1}}} .
\end{aligned}
$$

Due to the behaviour of $E_{2 n-1}^{-1} \approx n^{-2 / 3}$ for large values of $n$, as previously discussed, the double series in the denominator of (3.10) is convergent. In fact

$$
\begin{aligned}
& \sum_{n=1}^{\infty} \sum_{m=1}^{\infty} \frac{1}{E_{2 m-1}\left(E_{2 m-1}+E_{2 n-1}\right)^{2} E_{2 n-1}} \\
& \quad \leqslant \sum_{n=1}^{\infty} \sum_{m=1}^{\infty} \frac{1}{E_{2 m-1}^{2} E_{2 n-1}^{2}}=\left[\sum_{n=1}^{\infty} \frac{1}{E_{2 n-1}^{2}}\right]^{2}<\infty .
\end{aligned}
$$

Obviously, the operator in (3.10) is bounded since it is the sum of a bounded operator, the resolvent $\left(H_{0}^{p}-E\right)^{-1}$ plus a rank one operator. We still have to prove that this is indeed the resolvent of a self-adjoint operator. This proof is essentially the proof given in [33] in the study of the three-dimensional isotropic oscillator perturbed by a Dirac distribution, or in [1] in the discussion of the negative threedimensional Laplacian perturbed by a Dirac delta, so that we omit it.

Then, we have accomplished our purpose with a result that may be stated formally in the form of a theorem as follows:

Theorem 3.2. The bounded operator $\left(H_{\alpha}^{p}-E\right)^{-1}$, defined by the expression (3.10) is the resolvent of the self-adjoint Hamiltonian, which is usually given by the heuristic expression $H_{\alpha}^{p}=H_{0}^{p}-\mu_{\alpha}|\delta\rangle\langle\delta|$ with

$$
\begin{aligned}
\mu_{\alpha} & =\left[\sum_{n=1}^{\infty} \sum_{m=1}^{\infty} \frac{\psi_{2 m-1}^{2}(0) \psi_{2 n-1}^{2}(0)}{E_{2 m-1}+E_{2 n-1}}+\alpha\right]^{-1} \\
& =\left[\left(H_{0}^{p}\right)^{-1}(\mathbf{0}, \mathbf{0})+\alpha\right]^{-1} .
\end{aligned}
$$




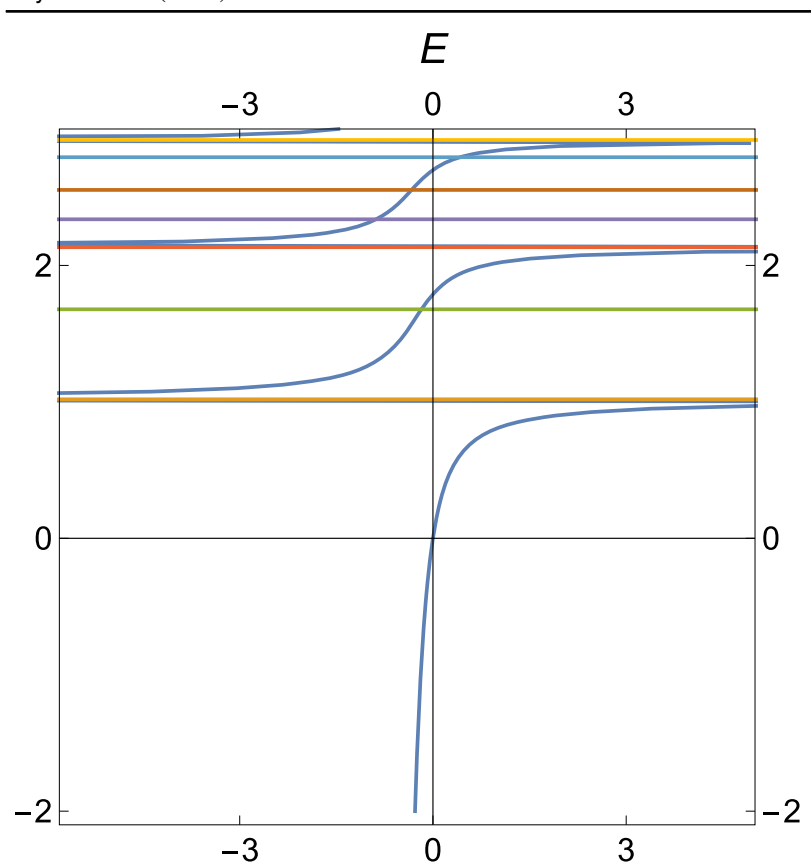

Figure 2. Plot of the three lowest eigenvalues (3.17) created by the point perturbation as functions of the extension parameter $\alpha$ (the horizontal lines show the eigenvalues of the unperturbed twodimensional operator with the pyramidal confinement).

Moreover, $H_{\alpha}^{p}$ regarded as a function of $\alpha$ is an analytic family in the sense of Kato.

Again, let us remark that $H_{\alpha=0}^{p} \neq H_{0}^{p}$ since $H_{\alpha=\infty}^{p}=H_{0}^{p}$, exactly as happened in the previous case.

As the two-dimensional pyramidal Hamiltonian $H_{0}^{p}$ does not have the same symmetry properties of the two-dimensional harmonic oscillator, we should expect that the properties of its eigenvalues are different in both cases. For the pyramidal case, the multiplicity of the eigenvalue $E_{n, m}=E_{n}+E_{m}$ of $H_{0}^{p}$ is equal to two if $n \neq m$ or one if the two indices coincide. In fact, each eigenvalue of the pyramidal $H_{0}^{p}$ with at least one even index, labelling an antisymmetric bound state of $h_{0}^{c}$, stays in the discrete spectrum of $H_{\alpha}^{p}$ with the same multiplicity, since the singular perturbation does not affect the corresponding subspace. On the contrary, the eigenvalues $E_{2 n+1,2 n+1}=E_{2 n+1}+E_{2 n+1}$ disappear from the spectrum, except when $\alpha=\infty$. Each eigenvalue $E_{2 n+1,2 m+1}=E_{2 n+1}+E_{2 m+1}, n \neq m$ of the pyramidal $H_{0}^{p}$ is still in the discrete spectrum of $H_{\alpha}^{p}$, although its multiplicity is lowered to one due to the emergence of a new eigenvalue. solutions of the equation:

$$
\begin{aligned}
\alpha & =\frac{E}{16} \sum_{n=1}^{\infty} \sum_{m=1}^{\infty} \\
& \times \frac{1}{E_{2 m-1}\left(E_{2 m-1}+E_{2 n-1}\right)\left(E_{2 m-1}+E_{2 n-1}-E\right) E_{2 n-1}} .
\end{aligned}
$$

In order to perform this summation, let us first write the expression under the sums as

$$
\begin{aligned}
& \frac{E}{E_{2 m-1}\left(E_{2 m-1}+E_{2 n-1}\right)\left(E_{2 m-1}+E_{2 n-1}-E\right) E_{2 n-1}} \\
& =\frac{1}{E_{2 m-1}\left(E_{2 m-1}+E_{2 n-1}-E\right) E_{2 n-1}} \\
& \quad-\frac{1}{E_{2 m-1}\left(E_{2 m-1}+E_{2 n-1}\right) E_{2 n-1}} .
\end{aligned}
$$

Then, (3.12) can be written as a sum of two terms We may use the results obtained in [32] so as to obtain the following expression for the second term in (3.13):

$$
\begin{aligned}
\frac{1}{16} & \sum_{n=1}^{\infty} \sum_{m=1}^{\infty} \frac{1}{E_{2 m-1}\left(E_{2 m-1}+E_{2 n-1}\right) E_{2 n-1}} \\
& =\sum_{n=1}^{\infty} \frac{1}{4 E_{2 n-1}}\left[\sum_{m=1}^{\infty} \frac{1}{4 E_{2 m-1}\left(E_{2 m-1}+E_{2 n-1}\right)}\right] \\
& =\sum_{n=1}^{\infty} \frac{\left(h_{0}^{c}+E_{2 n-1}\right)^{-1}(0,0)}{4 E_{2 n-1}} .
\end{aligned}
$$

Then, using the explicit expression of the Green function of the operator $h_{0}^{c}$ given in [32] and [47], the right hand side of (3.14) may be rewritten as a single summation involving a fraction having the Airy function in the numerator and its derivative in the denominator:

$$
\begin{gathered}
\sum_{n=1}^{\infty} \frac{1}{4 E_{2 n-1}}\left(h_{0}^{c}+E_{2 n-1}\right)^{-1}(0,0) \\
=-\sum_{n=1}^{\infty} \frac{1}{4 E_{2 n-1}} \frac{A i\left(2 E_{2 n-1}\right)}{A i^{\prime}\left(2 E_{2 n-1}\right)} .
\end{gathered}
$$

A similar manipulation may be performed in the first term of the second row in (3.13), so that (3.12) takes the following form:

$$
\alpha=\sum_{n=1}^{\infty} \frac{1}{4 E_{2 n-1}}\left[\frac{A i\left(2 E_{2 n-1}\right)}{A i^{\prime}\left(2 E_{2 n-1}\right)}-\frac{A i\left(2\left(E_{2 n-1}-E\right)\right)}{A i^{\prime}\left(2\left(E_{2 n-1}-E\right)\right)}\right]
$$

or equivalently,

$$
\alpha=\sum_{n=1}^{\infty} \frac{1}{4 E_{2 n-1}}\left[\frac{A i\left(2 E_{2 n-1}\right) A i^{\prime}\left(2\left(E_{2 n-1}-E\right)\right)-A i\left(2\left(E_{2 n-1}-E\right)\right) A i^{\prime}\left(2 E_{2 n-1}\right)}{A i^{\prime}\left(2 E_{2 n-1}\right) A i^{\prime}\left(2\left(E_{2 n-1}-E\right)\right)}\right] .
$$

The eigenvalues of $H_{\alpha}^{p}$ are the zeroes of the denominator in (3.10). In this pyramidal case, the new ground state energy and the new eigenvalues of the operator $H_{\alpha}^{p}$ are given by the
In opposition to the situation arising in the two-dimensional harmonic oscillator with a delta perturbation studied in the previous section, it does not seem possible to transform the 
series in (3.17) into an expression involving ordinary or special functions. This makes the study of this system more challenging, even in the investigation of the bound states. However, a lot of information can still be extracted from (3.17). First of all, it is possible to show that the sequence under summation in (3.17) behaves at infinity like $n^{-5 / 3}$. Once we have established this asymptotic behaviour, we make use of Mathematica in this study. Then, we may truncate the series after the first two thousand terms in order to obtain a relatively accurate description of the eigenvalues of $H_{\alpha}^{p}$ as functions of the extension parameter. This is shown in figure 2, where we show the behaviour of the three lowest energy values after the perturbation. this spectral structure is more complex than that of the perturbed two-dimensional isotropic harmonic oscillator due to the unequal spacing between each pair of adjacent energy eigenvalues of the unperturbed Hamiltonian $H_{0}^{p}$.

As can be seen, a level crossing takes place to the left of $\alpha=0$ between the second eigenenergy created by the point perturbation and the doubly degenerate eigenvalue $E_{1}+E_{2}=$ $E_{2}+E_{1} \approx 1.67845$, which is obviously unaffected by the perturbation. The eigenvalue $E_{1}+E_{3}=E_{3}+E_{1} \approx 2.13349$, depicted as the upper horizontal asymptote of the second eigenenergy created by the point perturbation, stays in the discrete spectrum even though it is no longer degenerate.

The third eigenenergy that emerged after the point perturbation shows three crossings with the unperturbed eigenvalues of $H_{0}^{p}$. While those with $2 E_{2} \approx 2.33811$ and $E_{1}+E_{4}=E_{4}+E_{1} \approx 2.55337$ occur to the left of $\alpha=0$, the one with $E_{2}+E_{3}=E_{3}+E_{2}$ takes place instead for a positive value of the extension parameter. Finally, the eigenvalue $E_{1}+E_{5}=E_{5}+E_{1} \approx 2.91945$, corresponding to the upper horizontal asymptote of the third eigenenergy created by the point perturbation, stays in the discrete spectrum even though it is no longer degenerate.

Alternatively, in total analogy with the previous section, figure 3 depicts the three lowest perturbed eigenvalues of $H_{1 / \beta}^{p}$, with $\beta=1 / \alpha$, as functions of the parameter representing the strength of the two-dimensional point interaction centred at the origin, as well as the right half of the fourth one.

\section{A mixed model: harmonic oscillator in one coordinate and conic in the other}

Finally, we reproduce the same discussion with the mixed Hamiltonian (1.3). Our resuls are similar to those obtained in sections 2 and 3. Proofs do not differ much from those needed in previous sections.

Theorem 4.1. The function $\left(H_{0}^{M}-E\right)^{-1}(\mathbf{x}, \mathbf{0})$ belongs to $L^{2}\left(\mathbb{R}^{2}\right)$ for any $\mathrm{E}$ in the resolvent set $\rho\left(H_{0}^{M}\right)$.

Proof. As in the previous cases, we just need to prove this result for $E<0$. Mimicking previous discussions, we have that

$$
\begin{aligned}
& \left\|\left(H_{0}^{M}-E\right)^{-1}(\mathbf{x}, \mathbf{0})\right\|_{2}^{2} \\
& =\sum_{n=0}^{\infty} \sum_{m=1}^{\infty} \frac{\phi_{2 n}^{2}(0) \psi_{2 m-1}^{2}(0)}{\left(2 n+\frac{1}{2}+E_{2 m-1}-E\right)^{2}} \\
& \leqslant \sum_{n=0}^{\infty} \sum_{m=1}^{\infty} \frac{\phi_{2 n}^{2}(0) \psi_{2 m-1}^{2}(0)}{\left(2 n+\frac{1}{2}+E_{2 m-1}\right)^{2}} \\
& \leqslant \frac{1}{4}\left[\sum_{n=0}^{\infty} \frac{\phi_{2 n}^{2}(0)}{2 n+\frac{1}{2}}\right]\left[\sum_{m=1}^{\infty} \frac{\psi_{2 m-1}^{2}(0)}{E_{2 m-1}}\right] .
\end{aligned}
$$

After (3.6), we know that the last series in (4.1) converges. The proof for the convergence of the other series is quite similar to that given in (2.3). In fact

$$
\begin{aligned}
& \sum_{n=0}^{\infty} \frac{\phi_{2 n}^{2}(0)}{2 n+\frac{1}{2}}=\frac{1}{\sqrt{\pi}} \int_{0}^{\infty} \frac{\mathrm{e}^{t / 2}}{\left(\mathrm{e}^{2 t}-1\right)^{1 / 2}} \mathrm{~d} t \\
& \quad=\frac{1}{\sqrt{\pi}} \int_{0}^{1} \frac{\xi^{-1 / 2}}{\left(1-\xi^{2}\right)^{1 / 2}} \mathrm{~d} \xi=\frac{\Gamma^{2}(1 / 4)}{2 \sqrt{2} \pi} .
\end{aligned}
$$

Our result is now proved.

We construct the resolvent following the recipe given in (2.4). In our case

$$
\left(H_{0}^{M}-E\right)^{-1}(\mathbf{0}, \mathbf{0})=\sum_{n=0}^{\infty} \sum_{m=1}^{\infty} \frac{\phi_{2 n}^{2}(0) \psi_{2 m-1}^{2}(0)}{2 n+\frac{1}{2}+E_{2 m-1}-E} .
$$

Following the regularisation procedure, (4.3) is to be replaced by

$$
\lim _{N \rightarrow \infty}\left[\sum_{n=0}^{N} \sum_{m=1}^{N} \frac{\phi_{2 n}^{2}(0) \psi_{2 m-1}^{2}(0)}{2 n+\frac{1}{2}+E_{2 m-1}-E}-\frac{1}{\mu(N)}\right],
$$

in the expression for the resolvent of $H_{\alpha}^{m}$ where

$$
\frac{1}{\mu(N)}=\sum_{n=0}^{N} \sum_{m=1}^{N} \frac{\phi_{2 n}^{2}(0) \psi_{2 m-1}^{2}(0)}{2 n+\frac{1}{2}+E_{2 m-1}} .
$$

This gives the following expression for the desired resolvent:

$$
\begin{aligned}
& \left(H_{\alpha}^{M}-E\right)^{-1}=\left(H_{0}^{M}-E\right)^{-1} \\
& \quad+\frac{\left|\left(H_{0}^{M}-E\right)^{-1}(\mathbf{x}, \mathbf{0})\right\rangle\left\langle\left(H_{0}^{M}-E\right)^{-1}(\mathbf{0}, \mathbf{y})\right|}{\alpha-E \sum_{n=0}^{\infty} \sum_{m=1}^{\infty} \frac{\phi_{2 n}^{2}(0) \psi_{2 m-1}^{2}(0)}{\left(2 n+\frac{1}{2}+E_{2 m-1}\right)\left(2 n+\frac{1}{2}+E_{2 m-1}-E\right)}} .
\end{aligned}
$$

In analogy with previous results drawn under similar circumstances, we obtain the following conclusion:

Theorem 4.2. The bounded operator $\left(H_{\alpha}^{M}-E\right)^{-1}$ is the resolvent of the self-adjoint Hamiltonian, which is usually given by the heuristic expression $H_{\alpha}^{M}=H_{0}^{M}-\mu_{\alpha}|\delta\rangle\langle\delta|$ with

$\mu_{\alpha}=\left[\sum_{n=0}^{\infty} \sum_{m=1}^{\infty} \frac{\phi_{2 n}^{2}(0) \psi_{2 m-1}^{2}(0)}{2 n+\frac{1}{2}+E_{2 m-1}}+\alpha\right]^{-1}, \quad \alpha \in \mathbb{R}$. 

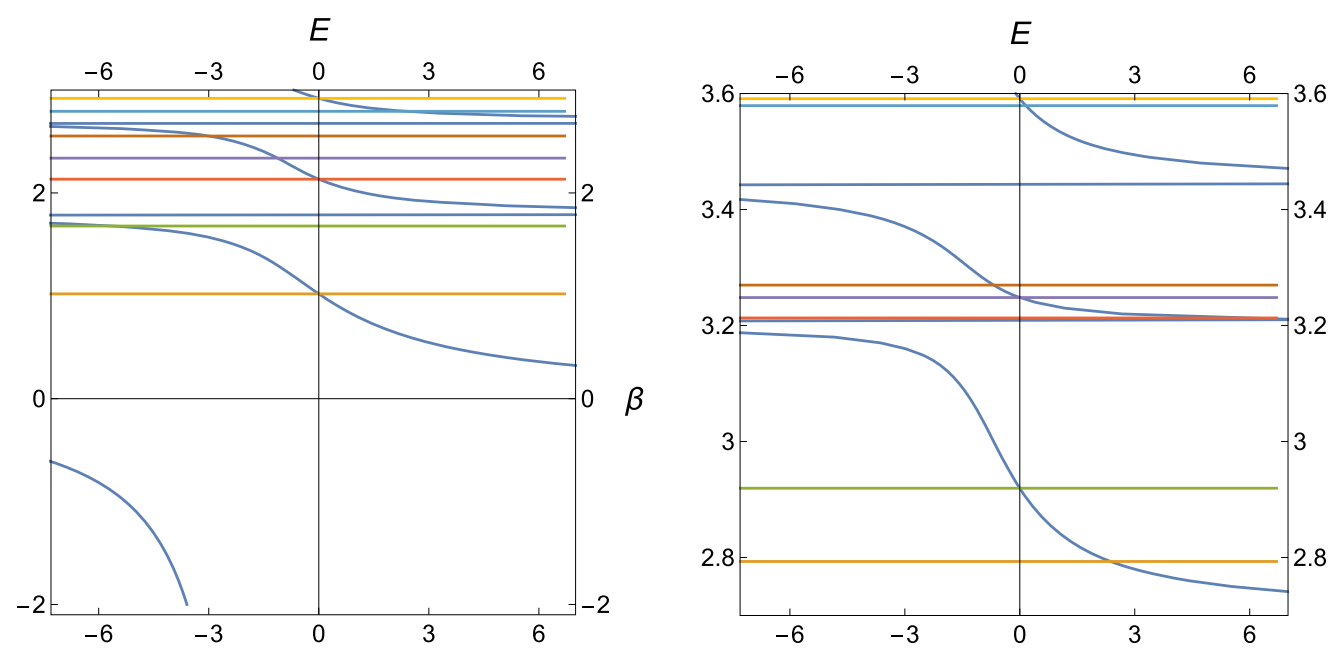

Figure 3. Left: Plot of the three lowest eigenvalues (3.17) created by the point perturbation as functions of the strength parameter $\beta=1 / \alpha$. Right: plot of the two next upper eigenvalues (3.17) created by the point perturbation as functions of the strength parameter $\beta=1 / \alpha$. In both figures, the horizontal lines show the eigenvalues of the unperturbed 2D operator with the pyramidal confinement.

Moreover, the family of Hamiltonians $H_{\alpha}^{M}$ is an analytic family with respect to $\alpha$ in the sense of Kato [1].

The eigenvalues of $H_{\alpha}^{M}$ are the real zeroes of the denominator in (4.6). This yields

$$
\begin{aligned}
\alpha= & E \sum_{n=0}^{\infty} \sum_{m=1}^{\infty} \frac{\phi_{2 n}^{2}(0) \psi_{2 m-1}^{2}(0)}{\left(2 n+\frac{1}{2}+E_{2 m-1}\right)\left(2 n+\frac{1}{2}+E_{2 m-1}-E\right)} \\
= & \sum_{n=0}^{\infty} \sum_{m=1}^{\infty} \frac{\phi_{2 n}^{2}(0) \psi_{2 m-1}^{2}(0)}{2 n+\frac{1}{2}+E_{2 m-1}-E} \\
& -\sum_{n=0}^{\infty} \sum_{m=1}^{\infty} \frac{\phi_{2 n}^{2}(0) \psi_{2 m-1}^{2}(0)}{2 n+\frac{1}{2}+E_{2 m-1}} \\
= & \sum_{n=0}^{\infty} \phi_{2 n}^{2}(0)\left[\sum_{m=1}^{\infty} \frac{\psi_{2 m-1}^{2}(0)}{2 n+\frac{1}{2}+E_{2 m-1}-E}\right. \\
& \left.-\sum_{m=1}^{\infty} \frac{\psi_{2 m-1}^{2}(0)}{2 n+\frac{1}{2}+E_{2 m-1}}\right] .
\end{aligned}
$$

Observe the expression between brackets in the last line in (4.8). It can be written in terms of the resolvent of the conic free Hamiltonian $h_{0}^{c}$ as defined in (3.1) calculated at the origin, see also (3.14). Then, (4.8) is equal to

$$
\begin{gathered}
\sum_{n=0}^{\infty} \phi_{2 n}^{2}(0)\left[\left(h_{0}^{c}+2 n+\frac{1}{2}-E\right)^{-1}(0,0)\right. \\
\left.-\left(h_{0}^{c}+2 n+\frac{1}{2}\right)^{-1}(0,0)\right] .
\end{gathered}
$$

As mentioned in section 3, the explicit expression for the resolvent of $h_{0}^{c}$ has been calculated in [32] and [47] and this result has already been used in (3.15). This shows that (4.9) is equal to

$$
\begin{aligned}
\alpha= & \sum_{n=0}^{\infty} \phi_{2 n}^{2}(0)\left[-\frac{A i(2(2 n+1 / 2-E))}{A i^{\prime}(2(2 n+1 / 2-E))}\right. \\
& \left.+\frac{A i(2(2 n+1 / 2))}{A i^{\prime}(2(2 n+1 / 2))}\right] \\
= & \frac{1}{\sqrt{\pi}} \sum_{n=0}^{\infty} \frac{(2 n-1) ! !}{2^{n} n !}\left[-\frac{A i(2(2 n+1 / 2-E))}{A i^{\prime}(2(2 n+1 / 2-E))}\right. \\
& \left.+\frac{A i(2(2 n+1 / 2))}{A i^{\prime}(2(2 n+1 / 2))}\right] .
\end{aligned}
$$

The expression between brackets in (4.10) behaves as $n^{-1}$ for $n$ large, so that the series in (4.10) converges since the other factor behaves like $n^{-1 / 2}$. As in the cases previously studied, we may represent graphically the dependence on either the parameter $\alpha$ or $\beta$ of the lowest energy levels using Mathematica. Then, the lowest energy levels are displayed in figure 4. As can be seen, the introduction of the conic confinement in one coordinate leads to the emergence of additional level crossings for the same perturbed eigenvalue to the right of the origin, that is to say when the perturbation becomes attractive.

\section{Concluding remarks}

Two-dimensional quantum systems have an interest of their own. In this paper, we have studied three solvable twodimensional quantum systems, the harmonic oscillator, the pyramidal potential and a combination thereof, all decorated with a contact perturbation in the form of a Dirac delta. In these cases, we have been able to show the behaviour of the energy for the lowest perturbed bound states in comparison with the energy of the same bound states before switching on the perturbation.

For the two-dimensional isotropic harmonic oscillator, if the energy levels of the unperturbed Hamiltonian $H_{0}^{o} E_{n, m}, n$ 

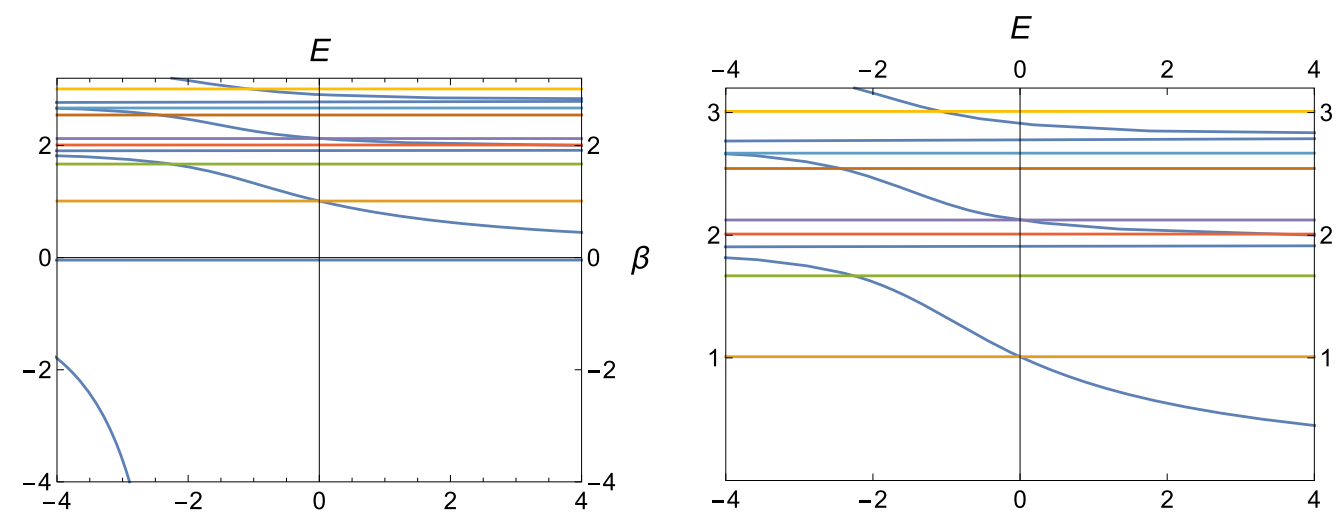

Figure 4. Plot of the three lowest eigenvalues (4.10) created by the point perturbation as functions of the strength parameter $\beta=1 / \alpha$.

or $m$ or both are odd, then, these are also energy levels for the perturbed Hamiltonian $H_{\alpha}^{o}$ with the same multiplicity. However, if both indices are even, $E_{2 n, 2 m}$ is still an eigenvalue of $H_{\alpha}^{o}$ although its multiplicity is reduced by one unit. Then, a new eigenvalue arises, corresponding the $s$-wave of $E_{2 n, 2 m}$, having a strong dependence on the parameter $\alpha$ as shown in figure 1 . This eigenvalue is smaller than the eigenvalue for the free Hamiltonian with the same indices $n$ and $m$ and approaches this value as $\alpha \rightarrow \infty$. For $n=m=0$, the eigenvalue of $H_{0}^{o}, E_{0,0}=1$, is no longer an eigenvalue of $H_{\alpha}^{o}$ and is replaced by a value of the energy which varies monotonically from 1 , at $\alpha=\infty$ to zero, at $\alpha=0$ taking negative values rapidly decreasing for $\alpha<0$. When the values of these two indices are even, say $2 n$ and $2 m$, the new eigenvalue for $H_{\alpha}^{o}$ has an energy equal to $E_{2 n, 2 m}=2 n+$ $2 m+1$ for $\alpha=\infty$, then decreases monotonically up to $2 n+2 m-1$ as $\alpha \rightarrow-\infty$. Obviously, it must cross the eigenvalue $2 n+2 m$ of $H_{0}^{o}$ at a certain value of $\alpha$, an effect we call energy level crossing. This situation is depicted in figure 1 for $n=0, m=2$, so that the total energy is 3 . We see that the eigenvalue of $H_{\alpha}^{o}$ crosses the value $E=2$ and tends asymptotically to $E=1$. These level crossings happen for any pair of even indices $2 n$ and $2 m$ and do not occur at the same value of $\alpha$.

For the pyramidal potential decorated with a delta at the origin, we obtain other results. Now, each energy level $E_{n, m}$ with at least one even index is not affected by the perturbation thus preserving its multiplicity. However, the eigenvalues of the form $E_{2 n+1,2 n+1}=E_{2 n+1}+E_{2 n+1}$ are no longer in the spectrum of $H_{\alpha}^{p}$, except for the obvious limit case $\alpha=\infty$. For $n \neq m$, the eigenvalue $E_{2 n+1,2 m+1}=E_{2 n+1}+E_{2 m+1}$ of the unperturbed pyramidal Hamiltonian $H_{0}^{p}$ is still an eigenvalue of $H_{\alpha}^{p}$, although with multiplicity lowered by one due to emergence of a new eigenvalue which strongly depends on $\alpha$. As in the harmonic oscillator case previously studied, this new eigenvalue is lower than $E_{2 n+1,2 m+1}$ and the ground state behaves exactly as in this case, where $E_{1,1}$ is not an eigenvalue of $H_{\alpha}^{p}$ except for $\alpha=\infty$. For others $E_{2 n+1,2 m+1}, n \neq m$, there are level crossings with lower energy values corresponding to $H_{0}^{p}$. These crossings are multiple and take place at different values of $\alpha$ with sharp contrast with the results obtained for the one-dimensional conic potential, of which the pyramidal potential represents a possible generalisation in the twodimensional case. Differently from the case of the perturbed two-dimensional harmonic oscillator, level crossings for $\beta>0$, i.e. when the perturbation becomes attractive, begin appearing for the same perturbed eigenvalue.

We have also investigated a mixed model, with the harmonic confinement in one coordinate and the conic confinement in the other. We have studied the behaviour of the lowest eigenvalues observing the the emergence of additional level crossings for the same perturbed eigenvalue to the right of $\beta=0$, that is to say when the perturbation becomes attractive.

Comparison between one-, two- and three-dimensional problems show substantial differences in the treatment of similar problems in all three cases and one obtains very different results [28]. These peculiarities emerging in twodimensional problems are interesting due to their possible applications to two-dimensional realistic systems such as graphene and a variety of thin films.

As possible extensions of this work we contemplate two situations. One is a generalisation of the pyramidal potential taking $V(x, y)=|x|^{\alpha}+|y|^{\beta}$, $\alpha$ and $\beta$ being real numbers. The other would correspond to a potential of the type $V(r)=-\alpha / \sqrt{r}$ with $r=\sqrt{x^{2}+y^{2}}$ studied in [39, 40]. Another direction is to use as perturbations extended singular distributions of $\delta(r-a)$ and $\delta^{\prime}(r-a)$ type. Work in this direction is in progress.

\section{Acknowledgments}

Stimulating discussions with Professor MJ López and Prof J Negro are kindly acknowledged. Partial financial support is acknowledged to the Spanish Junta de Castilla y León and FEDER funds (VA057U16) and MINECO (Project MTM2014-57129-C2-1-P). S Fassari and L Glasser wish to thank the entire staff at Departamento de Física Teórica, 
Atómica y Óptica, Universidad de Valladolid for their warm hospitality throughout their stays.

\section{Appendix. Some mathematical results}

Let us briefly comment some mathematical results concerning the free Hamiltonians of both cases object of our study. We begin with some definitions [48].

Let $B$ be a bounded operator on an infinite dimensional separable Hilbert space. Its singular values are the eigenvalues of the positive self-adjoint operator $|B|:=\sqrt{B^{\dagger} B}$, where $B^{\dagger}$ is the adjoint operator of $B$.

Let $s_{1} \geqslant s_{2} \geqslant \ldots \geqslant s_{n} \geqslant \ldots \geqslant 0$ be the singular values of $B$, then $B$ belongs to the Schatten class of index $p$ if and only if for some $p \in[1, \infty)$, we have that the following expression converges

$$
\|B\|_{p}:=\left(\sum_{n \geqslant 1} s_{n}^{p}\right)^{1 / p} .
$$

Operators in the Schatten class of index $p$ form a vector space over the complex numbers and $\|B\|_{p}$ is a norm on this space.

The operator $B$ belongs to the Schatten class of index $p$ if and only if $|B|^{p}$ is trace class. Then, $\|B\|_{p}^{p}=\operatorname{tr}\left(|B|^{p}\right)$.

After this brief introduction, let us give our results. First, we consider the Hamiltonian for the isotropic two-dimensional harmonic oscillator $H_{0}^{o}=\frac{1}{2}\left(-\Delta+x^{2}+y^{2}\right)$.

Theorem 6.1. The resolvent of the operator $H_{0}^{o}$ is a compact operator on $L^{2}\left(\mathbb{R}^{2}\right)$ belonging to the Schatten class of index $2+\epsilon, \epsilon>0$.

Proof. As a consequence of the first resolvent identity, it is sufficient to prove the statement only for any $E<0$. Hence, for any $E<0$ :

$$
\begin{aligned}
& \sum_{n_{1}, n_{2}=0}^{\infty} \frac{1}{\left(n_{1}+n_{2}+1-E\right)^{2+\epsilon}} \\
& \leqslant \sum_{n_{1}, n_{2}=0}^{\infty}\left[\frac{1}{\left(n_{1}+1\right)\left(n_{2}+1\right)}\right]^{1+\epsilon / 2} \\
& \quad=\left[\sum_{n=0}^{\infty} \frac{1}{(n+1)^{1+\epsilon / 2}}\right]^{2}<\infty .
\end{aligned}
$$

A similar result can be obtained when we instead have the free pyramidal Hamiltonian given by $H_{0}^{p}=\frac{1}{2}(-\Delta+|x|+|y|)$.

Theorem 6.2. The resolvent of the operator $H_{0}^{p}$ is a compact operator on $L^{2}\left(\mathbb{R}^{2}\right)$ belonging to the Schatten class of index $3+\epsilon, \epsilon>0$.

Proof. As a consequence of the first resolvent identity, we need only prove the statement for any $E<0$. Hence, for any

$$
\begin{aligned}
& E<0: \\
& \sum_{n=1}^{\infty} \sum_{m=1}^{\infty} \frac{1}{\left(E_{n}+E_{m}-E\right)^{3+\varepsilon}} \leqslant \sum_{n=1}^{\infty} \sum_{m=1}^{\infty} \frac{1}{\left(E_{n}+E_{m}\right)^{3+\varepsilon}} \\
& \quad \leqslant \sum_{n=1}^{\infty} \sum_{m=1}^{\infty} \frac{1}{E_{n}^{(3+\varepsilon) / 2} E_{m}^{(3+\varepsilon) / 2}}=\left[\sum_{n=1}^{\infty} \frac{1}{E_{n}^{(3+\varepsilon) / 2}}\right]^{2}<\infty,
\end{aligned}
$$

where we have used corollary 3.6 in [46], see also, [32, 38].

Similarly, in the case of the mixed model we have:

Theorem 6.3. The resolvent of the operator $H_{0}^{m}$ is a compact operator on $L^{2}\left(\mathbb{R}^{2}\right)$ belonging to the Schatten class of index $3+\epsilon, \epsilon>0$.

Proof. As a consequence of the first resolvent identity, we need only prove the statement for any $E<0$. Hence, for any $E<0$ :

$$
\begin{aligned}
& \sum_{n=0}^{\infty} \sum_{m=1}^{\infty} \frac{1}{\left(n+1 / 2+E_{m}-E\right)^{3+\varepsilon}} \\
& \quad \leqslant \sum_{n=0}^{\infty} \sum_{m=1}^{\infty} \frac{1}{\left(n+1 / 2+E_{m}\right)^{3+\varepsilon}} \\
& \quad \leqslant \sum_{n=0}^{\infty} \sum_{m=1}^{\infty} \frac{1}{(n+1 / 2)^{(3+\varepsilon) / 2} E_{m}^{(3+\varepsilon) / 2}} \\
& \quad \leqslant\left[\sum_{n=0}^{\infty} \frac{1}{(n+1 / 2)^{(3+\varepsilon) / 2}}\right]\left[\sum_{m=1}^{\infty} \frac{1}{E_{n}^{(3+\varepsilon) / 2}}\right]<\infty,
\end{aligned}
$$

where we have used corollary 3.6 in [46], see also, [32, 38].

\section{ORCID iDs}

S Fassari (ib https://orcid.org/0000-0003-3475-7696

M Gadella (i) https://orcid.org/0000-0001-8860-990X

F Rinaldi (iD https://orcid.org/0000-0002-0087-3042

\section{References}

[1] Albeverio S, Gesztesy F, Høegh-Krohn R and Holden H 2004 Solvable Models in Quantum Mechanics (AMS Chelsea Series) 2nd edn (Providence, RI: AMS)

[2] Albeverio S and Kurasov P 2000 Singular Perturbations of Differential Operators (Lecture Note Series) vol 271 (Cambridge: London Mathematical Society)

[3] Albeverio S, Fassari S and Rinaldi F 2013 J. Phys. A: Math. Theor. 46385305

[4] Albeverio S, Fassari S and Rinaldi F 2015 J. Phys. A: Math. Theor. 48185301

[5] Albeverio S, Fassari S and Rinaldi F 2016 J. Phys. A: Math. Theor. 49025302

[6] Erman F, Gadella M and Uncu H 2017 Phys. Rev. D 95 045004

[7] Erman F, Gadella M, Tunalı S and Uncu H 2017 Eur. Phys. J. Plus 132352

[8] Al-Hashimi M H, Shalaby A M and Wiese U J 2014 Phys. Rev. D 89125023 
[9] Gadella M, Glasser M L and Nieto L M 2011 Int. J. Theor. Phys. 50 2144-52

[10] Fassari S and Rinaldi F 2012 Rep. Math. Phys. 69 353-70

[11] Fassari S and Rinaldi F 2009 Rep. Math. Phys. 64 367-93

[12] Gadella M, Glasser M L and Nieto L M 2011 Int. J. Theor. Phys. 50 2191-200

[13] Gadella M, García-Ferrero M A, González-Martín S and Maldonado-Villamizar F H 2014 Int. J. Theor. Phys. 53 1614-27

[14] Zolotaryuk A V and Zolotaryuk Y 2015 J. Phys. A: Math. Theor. 48035302

[15] Zolotaryuk A V and Zolotaryuk Y 2015 Phys. Lett. A 379 511-7

[16] Gadella M, Heras F J H, Negro J and Nieto L M 2009 J. Phys. A: Math. Theor. 42465207

[17] Kulinskii V L and Panchenko D Y 2015 Physica B 472 78-83

[18] Seba P 1986 Czech. J. Phys. 36 667-73

[19] Bräunlich G, Hainzl C and Seiringer R 2013 Met. Funct. Anal. Top. 19 364-75

[20] Muñoz Castañeda J M, Mateos Guilarte J and Mosquera A M 2013 Phys. Rev. D 87105020

[21] Asorey M, García-Alvarez D and Muñoz Castañeda J M 2006 J. Phys. A: Math. Gen. $396127-36$

[22] Muñoz Castañeda J M, Kirsten K and Bordag M 2015 Lett. Math. Phys. 105 523-49

[23] Mateos Guilarte J and Castañeda J M Muñoz 2011 Int. J. Theor. Phys. 50 2227-41

[24] Bordag M 2014 Phys. Rev. D 89125015

[25] Gadella M, Mateos-Guilarte J, Muñoz Castañeda J M and Nieto L M 2016 J. Phys. A: Math. Theor. 49015204

[26] Asorey M and Muñoz Castañeda J M 2013 Nucl. Phys. B 874 852-76

[27] Antoine J P, Gesztesy F and Shabani J 1987 J. Math. Phys. 20 3687-712
[28] Fassari S, Gadella M, Glasser M L and Nieto L M 2018 Nanosyst., Phys., Chem., Math. 9 179-86

[29] Combescure M, Fayard C, Khare A and Richard J M 2011 J. Phys. A: Math. Theor. 44275302

[30] Farrel A and van Zyl B P 2010 J. Phys. A: Math. Theor. 43 015302

[31] Ferkous N 2013 Phys. Rev. A 88064101

[32] Fassari S, Gadella M, Glasser M L and Nieto L M 2018 Ann. Phys. 389 48-62

[33] Fassari S and Inglese G 1996 Helv. Phys. Acta 69 130-40

[34] Fassari S and Inglese G 1994 Helv. Phys. Acta 67 650-9

[35] Fassari S and Inglese G 1997 Helv. Phys. Acta 70 858-65

[36] Mityagin B S 2015 Int. J. Theor. Phys. 54 4068-85

[37] Mityagin B S and Siegl P 2016 Lett. Math. Phys. 106 $147-67$

[38] Fassari S, Gadella M, Glasser M L and Nieto L M 2017 (unpublished) arXiv: 1706.04916

[39] Li W-D and Dai W-S 2016 Ann. Phys. 373 207-15

[40] Fernández F F 2017 Ann. Phys. 379 83-5

[41] Bellandi Fo J and Caetano Neto E S 1976 J. Phys. A: Math. Gen. 9 683-5

[42] Reed M and Simon B 1972 Functional Analysis (New York: Academic)

[43] Reed M and Simon B 1979 Scattering Theory (New York: Academic)

[44] Albeverio S, Fassari S and Rinaldi F 2016 Nanosyst., Phys., Chem., Math. 7 803-15

[45] Albeverio S, Fassari S and Rinaldi F 2016 Nanosyst., Phys., Chem., Math. 7 268-89

[46] Lorinczi J and Malecki J 2012 J. Differ. Equ. 253 2486-871

[47] Glasser M L and Nieto L M 2015 Can. J. Phys. 93 1588-96

[48] Weidmann J 1980 Linear Operators in Hilbert Space (Berlin: Springer) 